\title{
Nuevas metodologías docentes para un nuevo mundo laboral
}

Peñaranda, David S. ${ }^{a^{*}}$, Naturil-Alfonso, Carmen ${ }^{a}$, Marco-Jiménez, Francisco ${ }^{a}$, y Vicente, José S. ${ }^{\text {a }}$

${ }^{a}$ Laboratorio de Biotecnología de la Reproducción, Departamento de Ciencia Animal, Universitat Politècnica de València. *Autor de correspondencia: dasncpea@upvnet.upv.es.

\begin{abstract}
The competences and skills to acquire by the student have evolved, so the educational methodology must also evolve. Society demands professionals with competences and skills in information and communication technologies (ICT), further a critical and deductive thinking.

The goal of this current paper is the description of a teaching project that proposes the implementation of flipped classroom in combination with ICT and learning stimulating methodologies, such as gamification. The teaching project will be applied in the subject: Animal and Human Physiology (Degree in Biotechnology, Universitat Piltècnica de València), which includes both "Alto Rendimiento Académico" (English group) and Spanish group.

Five activities have been designed to achieve the transversal competences selected for this subject. The teacher, based on the indicators of achievement and competence evidences, will be able to evaluate the effectiveness of the project, comparing the degree of achievement of the skills prior or postproject implementation. Furthermore, the final mark will also be compared with previous years, in order to test whether the innovative educational strategy has had a relevant impact on the final grade of the subject.
\end{abstract}

Keywords: flipped classrrom, ICT, gamification, teaching project

\footnotetext{
Resumen

Las competencias y habilidades a adquirir por parte del estudiante han evolucionado, por lo que la metodología educativa también debe evolucionar. La sociedad demanda profesionales con competencias y habilidades en tecnologías de la información y la comunicación (TIC) y que demuestren un pensamiento crítico y deductivo.

El objetivo del presente trabajo es la descripción de un proyecto docente que propone la implantación de la clase inversa en combinación con TIC y metodologías estimuladoras del aprendizaje, como la gamificación. El proyecto docente se aplicará en la asignatura de Fisiología Animal y Humana (Grado en Biotecnología; Universitat Piltècnica de València), tanto en el grupo Alto Rendimiento Académico (lengua vehicular inglés) como en un segundo grupo donde la lengua vehicular es el castellano.
} 
Han sido propuestas 5 actividades diseñadas para alcanzar las competencias transversales propuestas en la asignatura. El docente, basado en los indicadores de consecución y las evidencias de competencia, evaluará la efectividad del proyecto, comparando el grado de adquisición de las competencias previo o post-implantación del proyecto. Además, también se comparará la calificación final respecto a años anteriores, testando así si la innovadora estrategia educativa ha tenido un impacto en la nota final de la asignatura.

Palabras clave: clase invertida, TIC, gamificación, proyecto docente .

\section{Introducción}

El estilo de enseñanza tradicional persiste tanto en las escuelas primarias como secundarias, incluso a nivel de educación superior. De hecho, los estudiantes se sienten cómodos con este método, y la mayoría de ellos lo consideran una buena estrategia educativa (Thomasian, 2012). Con el objetivo de ofrecer un modelo de enseñanaza diferente, recientemente, se ha desarrollado un nueva metodología educativa: la clase inversa. Con esta metodología, los estudiantes pueden adquirir los resultados de aprendizaje fuera del aula, generalmente utilizando tecnologías de información y comunicación (TIC) como herramientas educativas en línea, screencast o videos grabados, mientras tanto, el tiempo de clase está reservado para la práctica, trabajo correctivo específico o actividades diseñadas para promover habilidades cognitivas de orden superior (Khan, 2012). Sin embargo, no todos los aspectos de la clase inversa son nuevos. En el marco de la educación tradicional, el docente también espera que los alumnos acudan al aula con cierta preparación previa, como haber leído o hecho material asignado (Davies et al., 2013). El elemento diferenciador reside en el gran avance de las herramientas TIC accesibles, tanto para estudiantes como para docentes (Davies y West, 2014), que permite que el aula invertida se haga realidad.

La forma de desarrollar la clase inversa puede variar, pero todos los modelos son básicamente los mismos (Talbert, 2012). El aprendizaje no se limita al aula, por lo que los estudiantes deben asumir la responsabilidad de su propio aprendizaje, ser más flexibles y adaptarse a esta metodología individualizada. Por otro lado, esta metodología educativa puede combinarse con otras tendencias de enseñanza innovadoras: como la adquisición de las competencias transversales. La sociedad contemporánea exige nuevas competencias tanto para docentes como para estudiantes, y estas competencias suelen estar relacionadas con actividades de aprendizaje electrónico, y por tanto con el uso de herramientas TIC. Aunque el uso de las TIC hace factible la implementación de la clase inversa, los investigadores han observado que la mayoría de los educadores aún adoptan pasivamente la tecnología como medio de aprendizaje (Wang et al., 2014). Estos resultados decepcionantes, han forzado a los educadores a reformar la enseñanza con el fin de mejorar los entornos de aprendizaje creativo. De esta forma, los alumnos son capaces de adquirir las competencias transversales a través de la resolución de problemas realistas y desarrollar habilidades cognitivas de orden superior (Lajoie y Azevedo, 2000). Para lograr este objetivo, la combinación de herraminetas TIC y la clase inversa pueden llegar a ser una

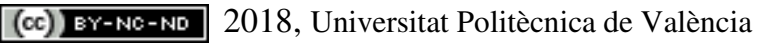

Congreso IN-RED (2018) 
metodología muy eficaz. El problema reside en que se requiere una alta formación, tanto por parte del docente como del estudiante, para el correcto uso de las TIC. Es por ello, que incluso en niveles de educación superior (universidad), la presencia de herramientas digitales y el desarrollo de competencias digitales es limitada. Hasta ahora, en la gran mayoría de los casos, las herramientas TIC se han utilizado de manera superficial, como en la planificación de lecciones, comunicación personal o procesamiento de textos (Drent y Meelissen, 2008), y la competencia digital de los alumnos se limita a habilidades básicas, incluidas herramientas de oficina y redes sociales (Valtonen et al., 2013).

Además de desarrollar las competencias transversales o adquirir la materia de la asignatura, la clase inversa tiene el objetivo de motivar a los estudiantes para el aprendizaje, y con este fin la clase inversa también puede ser combinada con estrategías educativas destinadas a este fin. Un ejemplo, sería la gamificación (Deterding et al., 2011; Huotari y Hamari, 2012). La gamificación ha sido implantada con resultados positivos en varios programas curriculares (Brewer et al., 2013; Fitz-Walter et al., 2011). Sin embargo, su implantación en la educación superior también ha sido limitada (Iosup y Epema, 2014), ya que si su evaluación no está bien equilibrada, puede inducir una disminución del interés en lugar de promover una motivación extra.

En conclusión, la clase inversa es una metodología educativa innovadora, lo suficientemente flexible como para incorporar otras estrategias de enseñanza como la gamificación o las TIC, facilitando el éxito en el logro de las competencias clave y transversales por parte del alumnado.

\section{Objetivo}

Aunque el objetivo del presente estudio es la descripción de un proyecto de enseñanza innovador a nivel de educación superior, el proyecto docente en si, presenta el siguiente objetivo:

- Trabajar y desarrollar tanto las competencias como las habilidades propopuestas para la asignatura "Fisiología Animal y Humana"

Para alcanzar el objetivo planteado, se implementarán nuevas metodologías docentes como la clase inversa y el uso de herramientas TIC.

\section{Desarrollo de la innovación}

\subsection{Descripción del ambiente de aprendizaje}

El proyecto docente se aplicará en la Universitat Politècnica de València (UPV), en el Grado en Biotecnología (http://www.upv.es/titulaciones/GB/index-en.html). El grado de Biotecnología de la UPV se encuentra en la primera posición del ranking de universidades españolas (http://www.elmundo.es/especiales/ranking-universidades/index.html), y la nota $\begin{array}{lllllll}\text { requerida de admisión es de } & 12.47 & \text { sobre } & 14\end{array}$ (https://www.upv.es/pls/oalu/sic_futuroalumno.notascorte?p_idioma=c). El grado se compone de 4 años académicos, incluyendo el trabajo final de grado (240 créditos del Sistema Europeo de Transferencia y Acumulación, ECTS). Además incluye el programa: Grupos de Alto Rendimiento Académico, grupos ARA 
(https://www.upv.es/contenidos/ARA/indexc.html). Este programa fue diseñado por el Generalitat Valenciana (http://www.ceice.gva.es/web/universidad/grupos-de-altorendimiento;\%20Generalitat\%20Valenciana) para reforzar el potencial de los estudiantes destacados con el fin de ayudarlos a lograr el mayor rendimiento académico.

El proyecto docente esta diseñado para implantarse en la asignatura anual: Fisiología Animal y Humana (código: 11109), correspondiente al segundo año académico, con 6 créditos ECTS de teoría y 3 de prácticas. La asignatura esta considerada como de formación básica, e incluye dos grupos: uno impartido en castellano y otro en inglés (ARA o grupo de alto rendimiento académico). Antes de asistir a este curso, los estudiantes deberían haber cursado las siguientes materias: Biología Celular, Química Biomolecular, Fundamentos de Química y Fundamentos de Física para Biotecnología. Por último, en el grupo ARA, para garantizar el logro de los resultados del aprendizaje, los estudiantes deben certificar que tienen un mínimo de nivel de inglés B2, según el Common European Framework of Reference for Languages (CEFR).

\subsection{Competencias y competencias transversales a adquirir}

El proyecto docente ha sido diseñado para lograr un conjunto de competencias:

- Capacidad de buscar y usar información

- Competencia para el aprendizaje continuo

- Capacidad para resolver problemas complejos

- Capacidad de evaluación crítica

- Trabajar en grupos multidisciplinarios

Con el objetivo de desarrollar estas competencias, se han definido los siguientes resultados de aprendizaje (Tabla 1). Por otro lado, todas las actividades han sido diseñadas para llevarse a cabo en grupos, desarrollando la competencia "Trabajo en grupos multidisciplinares".

Tabla 1. Competencias y resultados de aprendizaje

\begin{tabular}{|c|c|c|}
\hline & Competencias & Resultados de aprendizaje \\
\hline 1 & $\begin{array}{l}\text { Capacidad de buscar y usar } \\
\text { información }\end{array}$ & $\begin{array}{l}\text { Aplicar la regulación del Real Decreto para un caso práctico } \\
\text { de experimentación animal }\end{array}$ \\
\hline 2 & $\begin{array}{l}\text { Competencia para el } \\
\text { aprendizaje continuo }\end{array}$ & Ser capaz de realizar un diseño experimental \\
\hline 3 & $\begin{array}{l}\text { Competencia para el } \\
\text { aprendizaje continuo }\end{array}$ & Ser capaz de resumir y presentar un artículo de investigación \\
\hline 4 & Capacidad de evaluación crítica & Ser capaz de contrastar información usando herramientas TIC \\
\hline 5 & $\begin{array}{l}\text { Capacidad para resolver } \\
\text { problemas complejos }\end{array}$ & $\begin{array}{l}\text { Ser capaz de resolver problemas complejos mediante la } \\
\text { aplicación de los conocimientos adquiridos }\end{array}$ \\
\hline
\end{tabular}

(cc) EY-NC-ND 2018, Universitat Politècnica de València

Congreso IN-RED (2018) 


\subsection{Participantes y cronograma}

Antes de desarrollar el proyecto actual, la guía de enseñanza de Fisiología Animal y Humana incluía cinco seminarios, que consistían en dos presentaciones en el aula (trabajando en parejas) basadas en un tema específico o artículos científicos, además de 3 charlas en relación con la las últimas tecnologías de vanguardia relacionadas con la matería impartida.

Como se mencionó anteriormente, la asignatura incluye un grupo que usa el castellano como primer idioma y otro grupo que usa el inglés como idioma extranjero/segundo idioma. El grupo de inglés pertenece al programa ARA, que tiene un máximo de 25 estudiantes por aula. Por otro lado, el grupo de castellano está compuesto por alrededor de 100 estudiantes, divididos a su vez en cuatro grupos tanto para los seminarios como para las sesiones prácticas.

Las actividades docentes serán llevadas a cabo por un catedrático, un profesor titular y 2 profesores asociados, todos ellos pertenecientes al grupo de Laboratorio de Biotecnología Reproductiva, del Instituto de Ciencia y Tecnología Animal (ICTA) de la UPV. El proyecto ha sido diseñado para ser aplicado en 5 lecciones de 1 hora, correspondientes a los 5 seminarios descritos en la guía docente de la asignatura (https://gdocu.upv.es/alfresco/service/api/internal/shared/node/content/12lW_qRYQxadU_0 1JwgT3Q?a=true.pdf). Las actividades de enseñanza se llevarán desde de enero hasta mayo, y tendrán una periocidad mensual para cada uno de los grupos.

El material se entregará a través de la plataforma educativa en línea Poliforma $T$, que se basa en el sistema Sakai (http://asic.blogs.upv.es/tag/sakai/), siendo una herramienta TCI disponible tanto para estudiantes como para profesores. Esta plataforma educativa incluye una gran variedad de opciones, como una descripción de la guía de enseñanza, calendario, libro de calificaciones, anuncios y diferentes herramientas para comunicarse con los estudiantes: correo o chat (foros). Además, incluye una carpeta con recursos y una sección para realizar pruebas / cuestionarios y asignaciones. Finalmente, esta plataforma permitirá la entrega del material, como artículos científicos, guía de las diferentes actividades, material extra-material, screencasts o ejercicios. Por otro lado, los estudiantes también podrán subir sus posters, presentaciones o cualquier otra tarea solicitada. Se creará un foro para mejorar la comunicación, no solo con el responsable de la actividade sino también entre los compañeros de clase.

\section{4. • Desarrollo de la innovación}

Las actividades de enseñanza han sido diseñadas para alcanzar las competencias descritas con anterioridad. Todos ellos han sido organizados para ser realizados en grupos, con 5 miembros como máximo.

\subsubsection{Tarea 1: Experimentación animal en investigación}

Los animales son ampliamente utilizados en el campo de la biotecnología tanto para la investigación básica, desarrollo de fármacos, pruebas de toxicidad, fábricas 
biotecnológicas, etc. Sin embargo, todos estos procedimientos pueden causar dolor o sufrimiento en los animales, por lo que hay regulaciones estrictas para todos los estudios que incluyan experimentación animal. Como consecuencia, actualmente, no es posible solicitar un proyecto de investigación sin previa autorización por parte de un comité de ética independiente. Con el objetivo de introducir a los estudiantes en la regulación que concierne a la experimentación animal, se ha diseñado la siguiente actividad. Utilizando la plataforma Poliforma $T$, los alumnos tendrán acceso a las pautas y el material necesario para la realización de la actividad. Fuera del aula, los alumnos deberán rellenar un formulario tipo de experimentación animal basado en un caso real, el cual es proporcionado a los alumnos. En el formulario, los estudiantes deben describir el protocolo aplicado en el proyecto y confirmar si siguieron las normas de cuidado de la salud de los animales. Una vez en el aula, el profesor responsable de la actividad comentará los errores más comunes y las posibles dudas, y será posible abrir una discusión sobre la necesidad de regular la experimentación con animales.

Conocimiento a aprender: La regulación europea sobre experimentación animal

Habilidades a trabajar: Autonomía e iniciativa personal.

\subsubsection{Tarea 2: Realiza tu propio diseño experimental}

El diseño de experimentos que incluyen seres vivos es una responsabilidad y necesita conocimiento previo. Por tanto, una vez aprendido las reglas para realizar un diseño acorde al bienestar animal, los alumnos aprenderán a diseñar un experimento acorde criterios científicos. Para ello, los estudiantes diseñarán un experimento que incluirá un modelo animal siguiendo las pautas indicadas en la actividad. Para la realización de la actividad, tomarán como referencia artículos científicos que son accesibles tanto en papel como digitalmente en los servicios bibliográficos de la UPV. La actividad será entregada a través de la plataforma Poliforma $T$ y será se presentarda en el aula. Los profesores señalarán los posibles errores o dudas después de cada presentación.

Conocimiento a aprender: Aprender los principios básicos del diseño de un experimento que incluye seres vivos.

Habilidades a trabajar: Comunicación oral y creatividad.

\subsubsection{Tarea 3: Muestrame lo que sabes}

La capacidad de obtener la información más relevante y mostrarla de una manera atractiva es una tarea complicada. Con el objetivo de continuar con el aprendizaje progresivo, en la actividad actual los estudiantes deberán presentar los resultados del artículo científico utilizado en la actividad anterior, en un formato atractivo para llamar la atención a los demás científicos o al público en general. Para ello, los alumnos deberán elaborar un póster científico siguiendo los criterios descritos en la actividad fuera del aula, y una vez en el aula, realizarán una breve descripción de la investigación descrita en el póster. El responsable de la actividad señalará los posibles errores y mejoras.

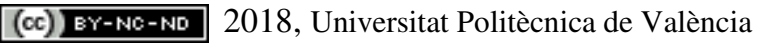

Congreso IN-RED (2018) 
Conocimiento a aprender: Aprender a obtener la información más relevante de un artículo científico.

Habilidades a trabajar: Síntesis, comunicación oral y creatividad.

\subsubsection{Tarea 4: No te creas todo lo lees}

Centrándonos en el mismo artículo científico de las dos actividades anteriores, en esta ocasión los alumnos deberán comprobar la veracidad de la discusión realizada, comprobando que las citas utilizadas son correctas e indicando que información relevante podría haberse incluido. Para ello, los alumnos subirán una presentación con el trabajo a la plataforma Poliforma T, y una vez en el aula realizarán una breve exposición.

Conocimiento a aprender: Aprender a contrastar información.

Habilidades a trabajar: Pensamiento crítico y habilidad para buscar información.

\subsubsection{Tarea 5: Encuentra quien es el culpable}

Una de las tareas más difíciles es aplicar la teoría para resolver un problema real. En la actividad actual, el profesor desarrollará un módulo de aprendizaje en la sección Lección de la plataforma Poliforma T. Este módulo de aprendizaje incluirá un screencast, más información adicional, en relación con los efectos de drogas o medicamentos. Luego, los estudiantes tendrán una actividad final con la descripción de una enfermedad. La tarea consistirá en descubrir cuáles son las causas de las enfermedades y qué órganos están involucrados.

Conocimiento a aprender: Aprender como las drogas/medicinas pueden afectar nuestro sistema nervioso.

Habilidades a trabajar: Pensamiento lógico y deductivo.

\subsection{Evaluación}

Las cinco actividades tendrán un peso del $15 \%$. Después de cada actividad y antes de la siguiente, el maestro entregará las calificaciones individuales para ser consultadas por cada alumno.

Con el objetivo de motivar a los estudiantes, el proyecto docente incluye una nueva metodología educativa: la gamificación. Imitando el ranking utilizado en las máquinas de videojuegos, los estudiantes se clasificarán según la marca que hayan obtenido después de cada actividad. Estableceremos tres categorías: el 33\% con las mejores calificaciones se colocará en la primera categoría, el siguiente $33 \%$ de las mejores calificaciones en la segunda categoría y el resto en la tercera categoría. Los estudiantes ubicados en la primera categoría obtendrán una puntuación extra de 0.5 puntos en la nota final de la asignatura, los estudiantes colocados en la segunda categoría de 0.25 puntos y los clasificados en la última no recibirán niguna puntuación extra.

La puntuación de cada actividad se realizará en base a los indicadores de consecución, los cuales se describen a continuación:

\subsubsection{Tarea 1: Experimentación animal en investigación}

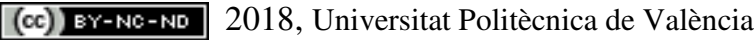

Congreso In-Red (2018) 
Descripción de los indicadores de consecución:

- Es capaz de identificar los puntos más relevantes del Real Decreto sobre experimentación animal.

- Sabe cómo aplicar la normativa descrita para la experimentación animal.

- Ha demostrado autonomía para desarrollar la actividad

La evidencia de la competencia será el formulario lleno entregado por el estudiante en la plataforma Poliforma T. Los alumnos deberán haber rellenado correctamente al menos un $40 \%$ del formulario para obtener una puntuación de aprobado ( 5 puntos) y más . completa $85 \%$ para obtener la máxima nota.

\subsubsection{Tarea 2: Realiza tu propio diseño experimental}

Descripción de los indicadores de consecución:

- Saber diseñar un experimento que se pueda llevar cabo según los criterios de bienestar y científicos especificados

- Conocer el procedimiento para diseño de un experimento que incluyacseres vivos.

- Mostrar creatividad

- Desarrollar habilidades orales

La evidencia de competencia será el documento del diseño experimental entregado por el alumno en la plataforma Poliforma T.

\subsubsection{Tarea 3: Muestrame lo que sabes}

Descripción de los indicadores de consecución:

- Habilidad de síntesis

- Mejorar la lectura comprensiva de artículos científicos escritos en una lengua extranjera (inglés)

- Mostrar creatividad

- Desarrollar habilidades orales

La evidencia de competencia será la presentación de los resultados en un formato de póster entregado por el alumno en la plataforma Poliforma T.

\subsubsection{Tarea 4: No te creas todo lo lees}

Descripción de los indicadores de consecución:

- Habilidad para buscar información

- Pensamiento crítico

- Capacidad de aplicar los conocimiento teóricos a casos prácticos

La evidencia de competencia será el documento, incluida la discusión de los resultados presentados en la tarea 3, y entregado por el alumno en la plataforma Poliforma T.

\subsubsection{Tarea 5: Encuentra quien es el culpable}

Descripción de los indicadores de consecución:

(c) EY-NC-ND 2018, Universitat Politècnica de València 
- Habilidad para resolver problemas complejos

- Capacidad de aplicar los conocimiento teóricos a casos prácticos

- Desarrollo del pensamiento lógico

- Mejora de la habilidaes deductivas

La evidencia de competencia será el documento que incluya la resolución del problema planteado, justificando la respuesta.

La evaluación de las diferentes competencias en cada actividad se llevará a cabo utilizando una nueva herramienta llamada iRubric, disponible en la plataforma Poliforma $\mathrm{T}$ (ejemplo: Tabla 2). Gracias a esta herramienta, iRubric, será posible evaluar las competencias desarrolladas en cada tarea usando una rúbrica específica, prediseñada para clasificar los resultados de aprendizaje en cada actividad.

Table 2. Ejemplo de iRubric para la evaluación de los estudiantes

\begin{tabular}{|c|c|c|c|c|c|c|c|}
\hline Categoría & 1 & 2 & 3 & 4 & Nota & Factor & $\begin{array}{c}\text { Nota } \\
\text { Defintiva }\end{array}$ \\
\hline Síntesis & $\begin{array}{c}\text { No cumple } \\
\text { con el } \\
\text { tiempo, } \\
\text { demasiados } \\
\text { datos } \\
\text { inútiles }\end{array}$ & $\begin{array}{l}\text { Cumple con } \\
\text { el tiempo, } \\
\text { pero } \\
\text { demasiada } \\
\text { información }\end{array}$ & $\begin{array}{c}\text { Cumple con el } \\
\text { tiempo y } \\
\text { muestra } \\
\text { (parcialmente) } \\
\text { los conceptos } \\
\text { más relevantes }\end{array}$ & $\begin{array}{c}\text { Cumple con el } \\
\text { calendario y } \\
\text { muestra (por } \\
\text { completo) los } \\
\text { conceptos más } \\
\text { relevantes }\end{array}$ & & $\mathrm{X} 2$ & \\
\hline Presentación & $\begin{array}{l}\text { Mal } \\
\text { estructurado } \\
\text { y difícil de } \\
\text { entender }\end{array}$ & $\begin{array}{c}\text { Bien } \\
\text { estructurado, } \\
\text { pero es } \\
\text { difícil de } \\
\text { seguir }\end{array}$ & $\begin{array}{c}\text { Bien } \\
\text { estructurado y } \\
\text { argumentado }\end{array}$ & $\begin{array}{c}\text { Bien } \\
\text { estructurado y } \\
\text { argumentado, } \\
\text { incluidos los } \\
\text { elementos que } \\
\text { lo hacen más } \\
\text { claro }\end{array}$ & & & \\
\hline Contentido & $\begin{array}{c}\text { No se ajusta } \\
\text { a lo } \\
\text { solicitado }\end{array}$ & $\begin{array}{c}\text { Se ajusta } \\
\text { parcialmente }\end{array}$ & $\begin{array}{c}\text { Coincide } \\
\text { correctamente } \\
\text { con lo que se } \\
\text { demandaba }\end{array}$ & $\begin{array}{c}\text { Coincide } \\
\text { perfectamente, } \\
\text { y además } \\
\text { incluye más } \\
\text { elementos que } \\
\text { dan un valor } \\
\text { extra }\end{array}$ & & X3 & \\
\hline $\begin{array}{c}\text { Expresión } \\
\text { oral }\end{array}$ & $\begin{array}{c}\text { No es capaz } \\
\text { de hablar en } \\
\text { público }\end{array}$ & $\begin{array}{l}\text { Muchos } \\
\text { errores }\end{array}$ & $\begin{array}{c}\text { Bien } \\
\text { presentado, } \\
\text { pero con } \\
\text { algunos } \\
\text { errores }\end{array}$ & $\begin{array}{c}\text { Bien } \\
\text { presentado, sin } \\
\text { errores }\end{array}$ & & & \\
\hline
\end{tabular}

\subsection{Conclusiones}

El objetivo de este proyecto docente es ayudar a los estudiantes a adquirir tanto las competencias como las habilidades propopuestas para la asignatura "Fisiología Animal y Humana" a través de la implementación de la clase inversa y el uso de herramientas TIC. El 
entorno donde se aplicará esta nueva metodología educativa serán en dos grupos docentes:: alumnos que usan el inglés o el español como lengua vehicular.

Para evaluar la efectividad del proyecto de enseñanza, compararemos la evaluación de competencias antes y después de la implantación del programa de enseñanza. Además, también se comparará la calificación final respecto a años anteriores, con el objetivo de observar si la nueva estrategia educativa ha tenido un impacto en los resultados de toda la asignatura, y no solo en parte relacionada con los seminarios.Finalmente, grado de satisfacción se evaluará a través de un cuestionario anónimo, en el que se les preguntará a los estudiantes qué actividades han sido más atractivas y qué aspectos cambiarán en el futuro.

Los resultados obtenidos serán presentados para ser difundidos en varias conferencias educativas, tales como:

- INRED 2018, IV Congreso Nacional de Innovación Educativa y Docencia en Red.

- $\quad 4^{\mathrm{a}}$ Conferencia Internacional sobre Avances en Educación Superior (HEAd'18).

\subsection{Referencias}

BREWER, R., ANTHONY, L., BROWN, Q., IRWIN, G., NIAS, J., y TATE, B. (2013). "Using Gamification to Motivate Children to Complete Empirical Studies in Lab Environments". In Proceedings of the 12th International Conference on Interaction Design and Children (pp. 388-391). New York, NY, USA: ACM. https://doi.org/10.1145/2485760.2485816

DAVIES, R. S., DEAN, D. L., y BALL, N. (2013). "Flipping the classroom and instructional technology integration in a college-level information systems spreadsheet course". Educational Technology Research and Development, Vol. 61(4), pp. 563-580. https://doi.org/10.1007/s11423-013-9305-6

SPECTOR, J. M., MERRILL, M. D., ELEN, J., y BISHOP, M. J., E.D. y DAVIES, R. S., y WEST, R. E. (2014). Technology Integration in Schools. In Handbook of Research on Educational Communications and Technology (pp. 841-853). New York, NY: Springer New York. https://doi.org/10.1007/978-1-4614-3185-5_68

DETERDING, S., DIXON, D., KHALED, R., y NACKE, L. (2011). "From game design elements to gamefulness: Defining gamification". Proceedings of the 15th International Academic MindTrek Conference on Envisioning Future Media Environments - MindTrek '11, 9-11. https://doi.org/10.1145/2181037.2181040

DRENT, M., y MEELISSEN, M. (2008). "Which factors obstruct or stimulate teacher educators to use ICT innovatively?" Computers y Education, Vol. 51(1), pp. 187199. https://doi.org/https://doi.org/10.1016/j.compedu.2007.05.001

FITZ-WALTER, Z., TJONDRONEGORO, D., y WYETH, P. (2011). "Orientation Passport: using gamification to engage university students". Proceedings of the 23rd Australian Computer-Human Interaction Conference on - OzCHI '11, 122-125. https://doi.org/10.1145/2071536.2071554

HUOTARI, K., y HAMARI, J. (2012). "Defining gamification". Proceeding of the 16th International Academic MindTrek Conference on - MindTrek '12, 17. https://doi.org/10.1145/2393132.2393137

IOSUP, A., y EPEMA, D. (2014). "An experience report on using gamification in technical higher education". Proceedings of the 45th ACM Technical Symposium on Computer

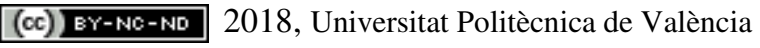

Congreso IN-RED (2018) 
Science Education - SIGCSE '14, (2008), 27-32. https://doi.org/10.1145/2538862.2538899

KHAN, S. (2012). The one world schoolhouse: Education reimagined. London: Hodder and Stoughton.

LAJOIE, S. P., y AZEVEDO, R. (2000). Cognitive tools for medical informatics. Computers as Cognitive Tools II: No More Walls: Theory Change, Paradigm Shifts and Their Influence on the Use of Computers for Instructional Purposes, 247-271.

THOMASIAN, J. (2012). Building a science, technology, engineering and math education agenda: an update of state actions. National Governors Association Center for Best Practices.

VALTONEN, T., HACKLIN, S., KONTKANEN, S., HARTIKAINEN-AHIA, A., KÄRKKÄINEN, S., y KUKKONEN, J. (2013). "Pre-service teachers' experiences of using social software applications for collaborative inquiry". Computers y Education, Vol. 69, pp. 85-95. https://doi.org/https://doi.org/10.1016/j.compedu.2013.07.001

WANG, S. K., HSU, H. Y., REEVES, T. C., y COSTER, D. C. (2014). "Professional development to enhance teachers' practices in using information and communication technologies (ICTs) as cognitive tools: Lessons learned from a design-based research study". Computers and Education, Vol. 79, pp. 101-115. https://doi.org/10.1016/j.compedu.2014.07.006

\subsection{Agradecimientos}

Este proyecto ha sido financiado por el Vicerrectorado de Estudios, Calidad y Acreditación de la Universidad Politécnica de Valencia (UPV) en el marco del Programa Proyectos de Innovación y Mejora Educativa (PIME/2017/B/010) y la Escuela de Ingeniería Agrícola y Medio Ambiente ( ETSIAMN) de la Universitat Politècnica de València (UPV). 